1ES 1553+113, and observed a high density of galaxies at the two redshifts associated with the absorption signals. Such densities are characteristic of the filamentary structures of the cosmic web. By combining the X-ray data with measurements of the ultraviolet emissions from 1ES 1553+113, Nicastro et al. estimated the density of the baryons associated with the X-ray absorbing features, and found that they account for $9-40 \%$ of the cosmic baryon density - suggesting that these features are a substantial reservoir of the missing baryons.

Weak X-ray absorption lines produced by baryons have been reported a few times before $^{7-8}$, but most of the results were marginal, and in some cases debatable. What is remarkable about the current work is that it represents the first time both of the expected absorption lines for helium-like oxygen have been detected together (for the absorption system at redshift 0.43 , although the statistical significance of one of the lines is marginal). The observation of two absorption lines from the same ion species is typically a good indication that the target ion species has been detected.

One concern is whether the observed X-rayabsorbing systems are truly located between Earth and 1ES 1553+113. The exact redshift of the BL Lac object is unknown; the best available estimate ${ }^{6}$ suggests that it is at least 0.41 . This value is less than the redshift of one of the X-ray absorbers, which implies that this absorber is either part of the BL Lac object, or a misidentification of something else. Nicastro et al. argue that both scenarios are unlikely, but an accurate measurement of the redshift of $1 \mathrm{ES} 1553+113$ is needed to resolve this issue.

It is also possible that the X-ray-absorbing systems are in galaxies, rather than in filamentary structures of the cosmic web - similar absorption systems have previously been detected in the Milky Way ${ }^{4}$. Nicastro and coworkers argue that this explanation is unlikely, partly because they did not find large galaxies similar to the Milky Way at the redshifts associated with the absorptions, but also because they did not detect any additional absorption lines from cold ions, which are typically found in galactic disks. These arguments are reasonable, but better observations are needed to rule out this scenario.

This type of observation, requiring more than a million seconds of exposure time, truly pushes the limits of the available instruments. Proposed space missions such as the Hot Universe Baryon Surveyor (go.nature. com/2luj4fa) and the Advanced Telescope for High-Energy Astrophysics (http://sci.esa.int/ athena/) will have much more sensitive X-ray spectrometers, and might eventually provide a complete map of the missing baryons in the cosmic web.

An alternative approach for detecting the missing baryons is to use a phenomenon known as the Sunyaev-Zel'dovich effect, in which high-energy electrons scatter off photons in the cosmic microwave background (CMB; electromagnetic radiation left over as a remnant of the Big Bang), thereby slightly distorting the CMB spectrum. High-energy electrons outside galaxies, and probably also in the filaments of the cosmic web, could produce such a distortion ${ }^{9}$, yielding a signal that indicates the presence of baryons. In the meantime, Nicastro and colleagues' findings offer a tantalizing glimpse of where the elusive missing baryons have been hiding.

Taotao Fang is in the Department of Astronomy and the Jiujiang Research Institute,
Xiamen University, Xiamen, Fujian 361005, China.

e-mail:fangt@xmu.edu.cn

1. Planck Collaboration. Astron. Astrophys. 594, 13 (2016).

2. Fukugita, M., Hogan, C. J. \& Peebles, P. J. E. Astrophys. J. 503, 518 (1998).

3. Cen, R. \& Ostriker, J. P. Astrophys. J. 514, 1 (1999)

4. Bregman, J. N. Annu. Rev. Astron. Astrophys. 45, 221-259 (2007)

5. Shull, J. M., Smith, B. D. \& Danforth, C. W. Astrophys. J. 759, 23 (2012).

6. Nicastro, F. et al. Nature 558, 406-409 (2018).

7. Fang, T., Marshall, H. L., Lee, J. C., Davis, D. S. \& Canizares, C. R. Astrophys. J. 572, L127 (2002).

8. Nicastro, F. et al. Nature 433, 495-498 (2005).

9. Hernández-Monteagudo, C. et al. Phys. Rev. Lett. 115, 191301 (2015).

\title{
CLINICAL ONCOLOGY
}

\section{Windows open for cancer immunotherapy}

Activating immune cells to destroy tumours is an effective strategy for treating an advanced lung cancer - but only for some people. Evidence that this approach has potential in early disease and as a combination therapy has now emerged.

\section{LIZZA E. HENDRIKS \& BENJAMIN BESSE}

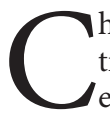
hemotherapy became the standard treatment for lung cancer in the twentieth century ${ }^{1}$. But in the past 15 years, there has been a drive to improve outcomes for people with this still-deadly disease, either through therapies that target enzymes encoded by genes harbouring cancer-driving mutations, or through immunotherapies, which activate the body's immune system to target tumours. Writing in The New England Journal of Medicine, two groups ${ }^{2,3}$ provide evidence that supports the use of immunotherapies to treat non-small-cell lung cancer (NSCLC) at different stages of the disease.

Tumour cells evade destruction by activating signals known as immune checkpoints, which deactivate immune cells called T cells ${ }^{4}$. Two immune checkpoints are the proteins cytotoxic T lymphocyte antigen 4 (CTLA-4) and programmed cell death 1 (PD-1), which are expressed by T cells themselves. Another, programmed cell death ligand 1 (PD-L1), is produced by tumour cells (Fig. 1).

Antibodies that interact with these proteins to prevent their normal activity, and so reawaken the immune system, are now used to treat metastatic NSCLC - the stage at which the cancer has spread. Antibodies that bind PD-1 or PD-L1 are sometimes successful in patients who have had treatments such as chemotherapy, but whose cancer has nonetheless progressed ${ }^{5}$. Alternatively, the anti-PD-1 antibody pembrolizumab can be used as a first-line treatment for metastatic
NSCLC when the percentage of tumour cells that express PD-L1 is high - these patients respond better to immunotherapy than to chemotherapy ${ }^{6}$.

If such immune-checkpoint-targeted antibodies (ICTs) can improve outcomes for metastatic NSCLC, could they also help to tackle early-stage disease? In the first of the current papers, Forde et al. ${ }^{2}$ carried out a pilot study to investigate whether the anti-PD-1 ICT nivolumab could be used to shrink tumours before surgery, which is a standard treatment for most cases of early-stage NSCLC.

The authors treated 21 patients with 2 doses of nivolumab 2 weeks apart, starting 4 weeks before the planned surgery date. They showed that surgery did not need to be delayed (for example, because of an adverse event with nivolumab) for any patient. The researchers anticipated that four weeks would not be enough time for the reactivated immune system to significantly shrink the tumour. Indeed, imaging revealed significant shrinkage in tumours in only two patients before surgery. However, examination of the surgically removed tumours revealed that $45 \%$ had undergone a major response to the ICT - less than $10 \%$ of the tumour cells remained alive. ICTs, unlike chemotherapy, cause inflammation and scar-tissue formation in tumours, and can therefore sometimes cause tumour growth. However, the researchers found that even two tumours that showed such growth had undergone a strong pathological response.

This level of efficacy is impressive, but needs to be further investigated by 


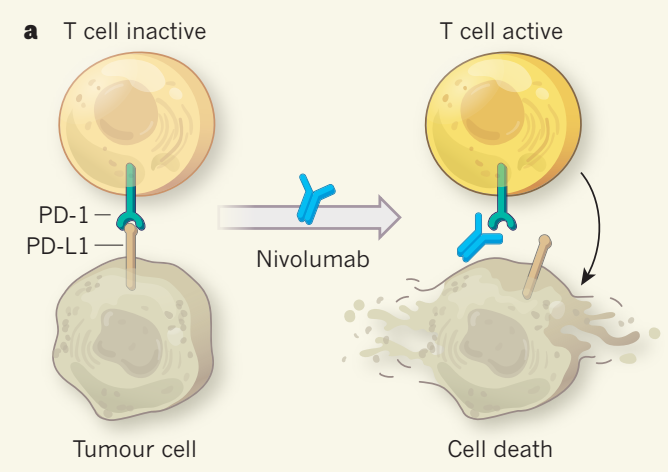

Figure 1 | Reawakening the immune system through different mechanisms. a, Tumour cells express the protein PD-L1 on their surfaces. PD-L1 binds to $\mathrm{PD}-1$ receptors on the surface of immune cells called T cells, inactivating the cells and so preventing them from targeting tumour cells for destruction. The antibody nivolumab prevents this interaction, and so reactivates T cells to destroy tumour cells. b, T cells can also be activated through interactions
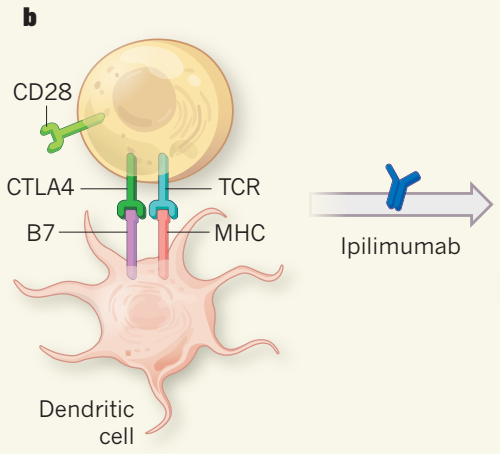

Ipilimumab

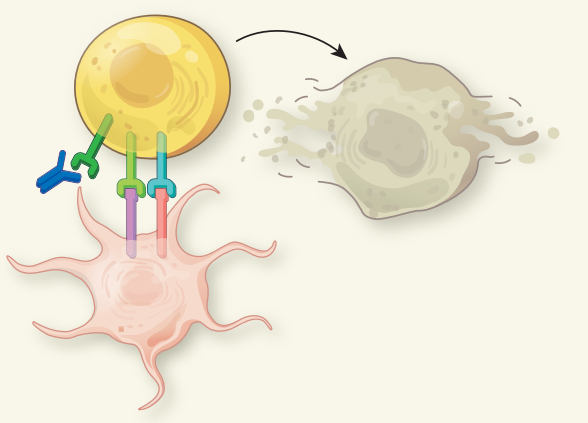

in-depth examination of the tumour specimens collected. Moreover, the optimal duration of nivolumab treatment remains to be determined, because delayed responses to ICTs can occur ${ }^{7}$. Of note, one of the patients enrolled experienced severe acute toxicity to nivolumab. Possible long-term side effects also need to be considered, because Forde et al. followed their patients for only a median of 12 months after surgery. Phase III trials are therefore now essential.

Forde and colleagues found that the number of mutations in each tumour's genome correlated with whether that tumour had a major pathological response to the ICT. And another study ${ }^{8}$ has found that a high number of mutations in a tumour - the tumour mutational burden (TMB) - is associated with an improved outcome in patients treated with a combination of ICTs. So, can TMB be used to predict which patients with advanced NSCLC would benefit from immunotherapy? In the second of the current papers, Hellmann et al. ${ }^{3}$ investigated this possibility, as part of a phase III trial called CheckMate 227.

The authors selected patients who had untreated, advanced-stage NSCLC. They assigned patients to one of four treatment groups - nivolumab; chemotherapy plus nivolumab; chemotherapy; or nivolumab plus the anti-CTLA4 antibody ipilimumab. For the last two groups, they analysed how a high TMB of at least 10 mutations per megabase of DNA affected progression-free survival - the time before the tumour begins to grow or spread once more.

Hellmann and colleagues found that median progression-free survival for patients who had a high TMB was 7.2 months for nivolumab plus ipilimumab, compared with 5.5 months for chemotherapy. After one year, there was no progression in $42.6 \%$ of patients who received the combination immunotherapy, compared with $13.2 \%$ of those who received chemotherapy alone. However, people with a low TMB did

not benefit from combination ICT. These results are remarkable - the first positive results for a combination ICT predicted using TMB.

What will the impact of these findings be, in an already crowded treatment field? One issue is the feasibility of using TMB to find suitable patients. Out of the 1,739 patients enrolled by Hellmann et al., only 1,004 could be evaluated for TMB, mainly because the amount of tissue available or the quality of the DNA extracted was inadequate. The analysis of progressionfree survival in patients with a high TMB involved only 299 people. Furthermore, there are multiple tests for TMB that analyse different

\section{"The authors found that the number of mutations in each tumour's genome correlated with whether that tumour had a major response to the immunotherapy."} genomic regions, and multiple cut-offs for high TMB classification - no cross-test validation exists. Other drawbacks of TMB analysis are the cost; the amount of tissue needed; and the fact that the analysis takes about two weeks. Finally, some of the tumours that had high TMB in Hellmann and colleagues' study also had high levels of PD-L1 expression. In these cases, single-agent immunotherapy is effective, and is less toxic than the combination treatment ${ }^{6}$.

It should be noted that two recently reported phase III trials ${ }^{9,10}$ found that the combination of chemotherapy and an ICT is superior to chemotherapy, whatever the level of PD-L1 expression (and possibly whatever the TMB). It is expected that these studies will establish a new standard of care. It will be hard for ICT combinations to compete.

ICTs can also have a dark side. One study ${ }^{11}$ found that $14 \%$ of people with NSCLC who were treated with ICTs developed hyperprogressive disease - an increase in tumour growth rate compared to the rate when patients were given a previous treatment. Hyperprogression is associated with poor survival rates. In the first few months of Hellmann and colleagues' trial, progression-free survival was higher for the chemotherapy arm than for the combination ICT, possibly suggestive of hyperprogression in some patients on the combination ICT. Whether such a pattern might arise in early-stage NSCLC, such as that examined by Forde et al., should also be carefully monitored. Finally, ICTs could harm our health-care systems through their cost more than US $\$ 10,000$ per month for a combination treatment ${ }^{12}$. We will need to monitor whether such a cost is sustainable.

Lizza E. Hendriks and Benjamin Besse are in the Department of Cancer Medicine, Institut d'Oncologie Thoracique, Gustave Roussy, 94805 Villejuif, France. L.E.H. is also in the Department of Pulmonary Diseases, Maastricht University Medical Center, the Netherlands. B.B. is also at the Université Paris Sud, Université Paris-Saclay. e-mails:lizza.hendriks@mumc.nl; benjamin.besse@gustaveroussy.fr

1. NSCLC Meta-Analyses Collaborative Group. J. Clin. Oncol. 26, 4617-4625 (2008)

2. Forde, P. M. et al. N. Engl. J. Med. 378, 1976-1986 (2018).

3. Hellmann, M. D. et al. N. Engl. J. Med. $\mathbf{3 7 8}$ 2093-2104 (2018).

4. Hoos, A. Nature Rev. Drug Discov. 15, 235-247 (2016)

5. Bianco, A., Malapelle, U., Rocco, D., Perrotta, F. \& Mazzarella, G. Curr. Opin. Pharmacol. 40, 46-50 (2018).

6. Reck, M. et al. N. Engl. J. Med. 375, 1823-1833 (2016).

7. Brahmer, J. et al. N. Engl. J. Med. 373, 123-135 (2015).

8. Hellmann, M. D. et al. Cancer Cell https://doi. org/10.1016/i.ccell.2018.03.018 (2018).

9. Gandhi, L. et al. N. Engl. J. Med. https://doi. org/10.1056/NEJMoa1801005 (2018).

10. Reck, M. et al. Ann. Oncol. 28 (Suppl. 11), xi31 (2017)

11.Ferrara, R. et al. J. Thorac. Oncol. 12 (Suppl. 2), S1843-S1844 (2017).

12.NICE. https://www.nice.org.uk/guidance/ta400 (2016).

This article was published online on 13 June 2018. 